\title{
On the Integrability of the Lie Algebra of the Conformal Group in Quantum Field Theory
}

\author{
Szymon Rabsztyn \\ Institute of Theoretical Physics, University of Wrocław, Wrocław, Poland
}

\begin{abstract}
It is shown that the infinitesimal conformal symmetry implies (in any quantum field theory which satisfies the Wightman axioms without invoking locality and global Poincaré symmetry) that there exists a uniquely defined unitary representation of the universal ( $\infty$-sheeted) covering group of the Minkowskian conformal group $\mathrm{SO}_{e}(4,2) /_{\mathbb{Z}_{2}}$. Proof was obtained using sufficient conditions for the integrability of a representation of a Lie algebra given by [8].
\end{abstract}

\section{Introduction}

Many papers have been written about global representations of the conformal group in the quantum field theories.

It has been first shown by [1-4] that global conformal transformations for free fields or generalized free fields can be defined without violation of causality.

Then Lüscher and Mack [5] proved that global Euclidean conformal symmetry of the Schwinger functions (weak conformal symmetry) implies that there exists a unique representation of the universal ( $\infty$-sheeted) covering group of the Minkowskian conformal group $S_{e}(4,2) / \mathbb{Z}_{2}$. In the same paper it has been stated also that a weak conformal symmetry implies the infinitesimal conformal symmetry of the fields.

The aim of the present paper is to prove that the infinitesimal conformal symmetry of the fields implies (in any quantum field theory which satisfies the Wightman axioms without invoking locality and global Poincare symmetry) that there exists a uniquely defined unitary representation of the universal ( $\infty$-sheeted) covering group of $S O_{e}(4,2) / \mathbb{Z}_{2}$. Our method is different from that used in the paper by Lüscher and Mack.

The problem is investigated in the following way: In Section 1 we present some facts concerning the mathematical background of the integrability problem.

The definition of a conformal quantum field theory is given in Section 2.

Finally, Section 3 deals with the problem of the integrability of the Lie algebra of the conformal group defined in Section 2.

Some details are included in the Appendix. 


\section{Preliminaries}

In this Section we shall collect some definitions and theorems without giving proofs.

They are either contained in given references or they are simple consequence of things established there.

Let $G$ be a finite dimensional real Lie group. The unitary (continuous) representation of $G$ in a Hilbert space $\mathscr{H}$ is the mapping $g \mapsto U(g), g \in G, U(g)$ unitary operator in $\mathscr{H}$, which satisfies the following conditions:

(i) for all $g_{1}, g_{2} \in G U\left(g_{1} g_{2}\right)=U\left(g_{1}\right) U\left(g_{2}\right)$,

(ii) $g \mapsto U(g)$ is continuous in the strong operator topology.

Let $\mathscr{G}$ be the Lie algebra of the Lie group $G$. For each $X \in \mathscr{G}$ the one-parameter subgroup, $t \mapsto \exp t X \in G, t \in \mathbb{R}$ is mapped (due to $U(g)$ ) into strongly continuous oneparameter group, $t \mapsto U(\exp t X)$ of the unitary operators.

From Stone's theorem follows the existence of the unique selfadjoint operator $d U(X)$ in $\mathscr{H}$ such that $U(\exp t X)=\exp i t d U(X)$ for each $t \in \mathbb{R}$.

Theorem 1. For each unitary representation of $G$ in $\mathscr{H}$ there exists a dense set $\mathscr{D}_{U} \subset \mathscr{H}$ for which $d U(X)$ is defined, essentially selfadjoint for all $X \in \mathscr{G}$ (i.e. $\left.\left(d U(X) \uparrow \mathscr{D}_{U}\right)^{*}=\left(d U(X) \uparrow \mathscr{D}_{U}\right)^{* *}=d U(X)\right)$ and $d U(X) \mathscr{D}_{U} \subset \mathscr{D}_{U}$. Moreover, id $U(X)$ satisfy on $\mathscr{D}_{U}$ the linear and commutation relations of the underlying Lie algebra $\mathscr{G}$.

Proof. See [6].

We may generalize the scheme above and define a symmetrical representation of the Lie algebra $\mathscr{G}$ on a dense set $\mathscr{D} \subset \mathscr{H}$ as the mapping of $\mathscr{G} \ni X \mapsto T(X) \in L(\mathscr{D}, \mathscr{D})$ into the set of linear operators defined on $\mathscr{D}$, with the range in $\mathscr{D}$, which satisfies:

(i) $\left(T(X) \Psi_{1}, \Psi_{2}\right)=\left(\Psi_{1}, T(X) \Psi_{2}\right)$, for each $\Psi_{1}, \Psi_{2} \in \mathscr{D}, X \in \mathscr{G}$,

(ii) $T(\lambda X+\mu Y)=\lambda T(X)+\mu T(Y)$, for each $\lambda, \mu \in \mathbb{R}$,

(iii) $i T([X, Y])=T(X) T(Y)-T(Y) T(X)$, for each $X, Y \in \mathscr{G}$.

We shall say that a symmetrical representation of the Lie algebra $\mathscr{G}$ on $\mathscr{D}$ is integrable (resp. uniquely integrable), if there exists in $\mathscr{H}$ a (resp. only one) unitary representation of the connected and simply connected Lie group $G$, with the Lie algebra $\mathscr{G}$ such that

$$
d U(X) \uparrow \mathscr{D}=T(X) .
$$

We call a vector $\Psi$ analytic (resp. semi-analytic) for an operator $A$ if $\Psi \in \bigcap_{n=1}^{\infty} \mathscr{D}\left(A^{n}\right)$ and $\sum_{n=0}^{\infty}\left(t^{n} / n !\right)\left\|A^{n} \Psi\right\|\left(\right.$ resp. $\sum_{n=0}^{\infty}\left(t^{n} /(2 n) !\left\|A^{n} \Psi\right\|\right)$ converges for certain $t>0$. A vector $\Psi \in \mathscr{H}$ is an analytic vector for the representation $U(g)$ of the Lie group $G$ in $\mathscr{H}$ if the mapping $g \mapsto U(g) \Psi$ defined on $G$ is an analytic vector valued function (for the definition see Appendix 1). Since translations by elements of $G$ are analytic isomorphisms, $g \mapsto U(g) \Psi$ is analytic on all $G$ iff it is analytic in a neighborhood of the group unity.

A set of vectors $Y_{1}, \ldots, Y_{m}$ (resp. $\left.X_{1}, \ldots, X_{n}\right)$ in $\mathscr{G}$ is a set of generators of $\mathscr{G}$ (resp. basis of $\mathscr{G}$ ) if $\mathscr{G}$ is generated by linear combinations of the vectors

$$
Y_{1}, \ldots, Y_{m}, \quad\left[Y_{a_{1}}, Y_{a_{2}}\right], \quad\left[Y_{a_{1}},\left[Y_{a_{2}}, Y_{a_{3}}\right]\right], \ldots \quad 1 \leqq a_{1}, a_{2}, \ldots \leqq m
$$

(resp. $X_{1}, \ldots, X_{n}$ ). 
Theorem 2. Given a symmetrical operator A defined on $\mathscr{D}(A)$ (which is bounded from below). If $\mathscr{D}(A)$ contains a total set-i.e. a set for which the linear hull is dense - of analytic (resp. semi-analytic) vectors for $A$ then $A$ is essentially selfadjoint.

Proof. See [7].

Theorem 3. Let $T(X)$ be a symmetrical representation of a finite dimensional real Lie algebra $\mathscr{G}$ on $\mathscr{D}$. If there exists a basis in $\mathscr{G}, X_{1} \ldots, X_{n}$, such that we have in $\mathscr{D}$ a total set each vector of which is analytic for $T\left(X_{a}\right), a=1, \ldots, n$, then the representation is uniquely integrable to a unitary representation of the connected and simply connected Lie group, the Lie algebra of which is $\mathscr{G}$ (see Appendix 4).

Proof. This follows directly from the work $[8,9]$.

As an immediate consequence of the definition of a set of generators we have the

Lemma. If $T(X)$ is a symmetrical representation on $\mathscr{D}$ of the Lie algebra $\mathscr{G}, T(\mathscr{G})$ is integrable and there exists a set of generators such that $T\left(Y_{a}\right), a=1, \ldots, m$, are essentially selfadjoint on $\mathscr{D}$, then the representation is uniquely integrable.

In what follows we shall denote by $A$ the quantity $T\left(X_{a}\right)$.

\section{Assumptions-Formulation of a Conformal Quantum Field Theory}

To simplify the notation and make the calculations more clear we shall consider a theory of one scalar neutral field in four dimensions.

A finite set of fields with spin in any dimensions $\geqq 2$ can be consider without changing the validity of the results.

We shall assume the following Axioms, which defined a conformal quantum field theory:

A0. The space of states is a separable Hilbert space $\mathscr{H}$ over the complex field $\mathbb{C}$ with dinstinguished vector $\Omega$-vacuum state.

A1. There exists a linear subset $\mathscr{D} \subset \mathscr{H}, \Omega \in \mathscr{D}$ and a linear mapping $\phi: \mathscr{S}\left(\mathbb{R}^{4}\right) \rightarrow L(\mathscr{D}, \mathscr{D})$ such that

$$
\left(\Psi_{1}, \phi(f) \Psi_{2}\right) \in \mathscr{S}^{\prime}\left(\mathbb{R}^{4}\right), \quad\left(\phi(f) \Psi_{1}, \Psi_{2}\right)=\left(\Psi_{1}, \phi(\bar{f}) \Psi_{2}\right)
$$

for each $\Psi_{1}, \Psi_{2} \in \mathscr{D}, f \in \mathscr{S}\left(\mathbb{R}^{4}\right)$.

$A 2$. The set $\mathscr{D}_{0}:=\operatorname{Lin}\left\{\phi\left(f_{1}\right) \ldots \phi\left(f_{k}\right) \Omega ; f_{i} \in \mathscr{S}\left(\mathbb{R}^{4}\right), k=0,1, \ldots\right\}$ is dense in $\mathscr{H}$.

$A 3$. There exist fifteen operators $P^{\mu}, M^{\mu \nu}, D, K^{\mu}, \mu=0,1,2,3$ defined and symmetrical on $\mathscr{D}_{0}$, with the range in $\mathscr{D}$ which satisfy the following conditions:

a) $P^{\mu} \Omega=0$

$$
\left.\begin{array}{l}
M^{\mu \nu} \Omega=0 \\
{\left[P^{\mu}, \phi(f)\right]=\phi\left(i \partial^{\mu} f\right)} \\
{\left[M^{\mu \nu}, \phi(f)\right]=\phi\left(i\left(x^{\mu} \partial^{v}-x^{v} \partial^{\mu}\right) f\right)}
\end{array}\right\} \text { on } \mathscr{D}_{0},
$$

b) $D \Omega=0$

$[D, \phi(f)]=\phi\left(i\left(x^{\mu} \partial_{\mu}+4-d\right) f\right)$ on $\mathscr{D}_{0}$

c) $\left(\Psi, P^{0} \Psi\right) \geqq 0$ for each $\Psi \in \mathscr{D}_{0}$,

d) $K^{\mu} \Omega=0$

$$
\left[K^{\mu}, \phi(f)\right]=\phi\left(i\left(2 x^{\mu} x^{v} \partial_{v}-x^{2} \partial^{\mu}+2 x^{\mu}(4-d)\right) f\right) \text { on } \mathscr{D}_{0} \text {. }
$$


By straightforward calculation it can be checked that $A \mathscr{D}_{0} \subset \mathscr{D}_{0}$, where $A=P^{\mu}, M^{\mu \nu}, D, K^{\mu}$ and the following equalities are satisfy on $\mathscr{D}_{0}$ :

$$
\begin{aligned}
{\left[M^{\varrho \sigma}, M^{\mu \nu}\right] } & =i\left(g^{\varrho v} M^{\sigma \mu}+g^{\sigma \mu} M^{\varrho v}-g^{\varrho \mu} M^{\sigma v}-g^{\sigma \nu} M^{\varrho \mu}\right) \\
{\left[P^{\mu}, M^{\varrho \tau}\right] } & =i\left(g^{\mu \varrho} P^{\tau}-g^{\mu \tau} P^{\varrho}\right) \\
{\left[P^{\mu}, P^{\nu}\right] } & =0 \\
{\left[D, P^{\mu}\right] } & =-i P^{\mu} \\
{\left[D, M^{\mu \nu}\right] } & =0 \\
{\left[D, K^{\mu}\right] } & =i K^{\mu} \\
{\left[K^{\lambda}, P^{\mu}\right] } & =-2 i\left(g^{\lambda \mu} D+M^{\lambda \mu}\right) \\
{\left[K^{\lambda}, K^{\mu}\right] } & =0 \\
{\left[K^{\lambda}, M^{\mu \nu}\right] } & =i\left(g^{\lambda \mu} K^{v}-g^{\lambda \nu} K^{\mu}\right) .
\end{aligned}
$$

Thus we have on $\mathscr{D}_{0}$ the symmetrical representation of the Lie algebra of the conformal group.

Here the conformal group means the connected and simply connected Lie group with the Lie algebra which satisfies the above equalities with -1 replacing $i$ - universal covering group of e.g., $S U(2,2)[10], S O_{e}(4,2) /_{\mathbb{Z}_{2}}$.

The question arises:

Is this representation integrable and if so-is it uniquely integrable?

\section{Results}

We are going to show that the representation of the Lie algebra of the conformal group defined in Section 2 is uniquely integrable.

We present first some results about integrability of the Lie algebra of the Weyl group.

To this aim let us introduce, following Snellman [11], the set

$$
\mathscr{D}_{0}^{\prime}:=\left\{\Psi \in \mathscr{H} ; \Psi=\phi\left(f_{\left\{n_{1}\right\}}\right) \ldots \phi\left(f_{\left\{n_{k}\right\}}\right) \Omega, \Psi=c \Omega, k=1,2 \ldots\right\} ;
$$

where

$$
f_{\{n\}} \in H\left(\mathbb{R}^{4}\right)
$$

the space of Hermite functions in four dimensions i.e.

$$
f_{\{n\}}(x)=f_{n_{0}}\left(x^{0}\right) \ldots f_{n_{3}}\left(x^{3}\right), \quad f_{n_{\mu}}\left(x^{\mu}\right) \in H(\mathbb{R}) .
$$

From $A 2$ the linear hull of $\mathscr{D}_{0}^{\prime}$ is dense in $\mathscr{H}$ because evidently $\mathscr{D}_{0}^{\prime}$ is total in $\mathscr{D}_{0}$. We have the theorem which is a generalization of the theorem of Snellman [11].

Theorem. If $A 0,1,3 a, 3 b$ are satisfied, then every $\Psi \in \mathscr{D}_{0}^{\prime}$ is an analytic vector for an arbitrary linear combination of $P^{\mu}, M^{\mu \nu}, D$.

Proof. See Appendix 2.

From Theorem 2,3 of Section 1 follows 
Corollary. If Axioms A0,1,2,3a,b are satisfied the Lie algebra of the Weyl group is uniquely integrable to a unitary representation of the Weyl group. The operators $P^{\mu}, M^{\mu v}, D$ are essentially selfadjoint on $\mathscr{D}_{0}$.

Using results of [9], Theorem 3 (see also [11]), we have the

Corollary. Every $\Psi \in \mathscr{D}_{0}^{\prime}$ is an analytic vector for the representation of the Weyl group.

Thus we can assume instead of $A 3 a, b, c$ equivalently:

$A 3^{\prime} a, b, c$ (resp. $\left.A 3^{\prime} a, c\right)$. There exists a continuous unitary representation of the Weyl (resp. Poincaré) group on $\mathscr{H}$ such that

$$
\begin{aligned}
& U_{d}(a, \Lambda, \lambda) \Omega=\Omega, \quad U_{d}(a, \Lambda, \lambda) \mathscr{D}_{0} \subset \mathscr{D}_{0} \\
& U_{d}(a, \Lambda, \lambda) \phi(f) U_{d}(a, \Lambda, \lambda)^{*}=\phi\left(\lambda^{4-d} f\left(\lambda^{-1} \Lambda^{-1}(x-a)\right)\right)
\end{aligned}
$$

(resp. $\lambda \equiv 1$ ), and the generators of translations $P^{\mu}$ have their spectral support in $\bar{V}^{+}$.

So far the operators constituting the symmetrical representation of the Lie algebra of the conformal group were defined only on $\mathscr{D}_{0}$. This is not convenient to prove integrability.

Taking into account the lemma from Appendix 3 we can define some symmetrical extension of the representation defined in Section 2 from $\mathscr{D}_{0}$ to $\mathscr{D}_{z, 0}$ (see Appendix 3 for the notation).

Namely we define

$$
\mathscr{A} \Psi(f):=\Psi\left(\sum_{i=1}^{k} a_{i} f\right), \quad a_{i}=W_{a}^{d}\left(x_{i}^{0}, \ldots, x_{i}^{3}, \partial_{0}^{i}, \ldots, \partial_{3}^{i}\right),
$$

where $W_{a}^{d}, a=1, \ldots, 15$, is one from the fifteen differential operators from the right hand side equalities $A 3 a, b, d, \Psi(f)$ is "an extension" of $\phi\left(f_{1}\right) \ldots \phi\left(f_{k}\right) \Omega$, and

$$
\mathscr{A} \tilde{R}_{k} \Psi\left(z_{1}, \ldots, z_{k}\right):=\sum_{i=1}^{k} \tilde{R}_{k} \tilde{a}_{i} \Psi\left(z_{1}, \ldots, z_{k}\right),
$$

where

$$
\tilde{a}_{i}=W_{a}^{4-d}\left(z_{i}^{0}, \ldots, z_{i}^{3},-\partial_{0}^{i}, \ldots,-\partial_{3}^{i}\right),
$$

$\Psi\left(z_{1}, \ldots, z_{k}\right)$ is "an extension" of $\Psi(f), \tilde{R}_{k}$ is an operator polynomial in $z_{i}^{\mu}, \partial / \partial z_{i}^{\mu}$, $\mu=0,1,2,3, i=1, \ldots, k$.

By straightforward calculation one can check the

Lemma. This new operators form the symmetrical representation of the Lie algebra of the conformal group which coincides with the former one on $\mathscr{D}_{0}$ (for details see Appendix 3).

The new representation is also an extension of the representation on $\mathscr{D}_{z}$ which we obtain by the restriction $\mathscr{A}$ to $\mathscr{D}_{z}$.

We have the

Theorem. Each vector $\Psi \in \mathscr{D}_{z}$ is an analytic vector for $\mathscr{A}$.

Proof. Consider $\Psi\left(z_{1}, \ldots, z_{k}\right)$ with fixed $\left(z_{1}, \ldots, z_{k}\right) \in Z(k)$. 
Lemma. We can take such an open neighbourhood of the unity $O\left(z_{1}, \ldots, z_{k}\right)$ in the conformal group $G$ and such a parametrization that:

(i) $\left(g z_{1}, \ldots, g z_{k}\right) \in Z(k)$ for $g \in O\left(z_{1}, \ldots, z_{k}\right)$ and $\left(g z_{1}, \ldots, g z_{k}\right)$ is the analytic function of the parameters of the group. This analytic function is defined for $g \in O\left(z_{1}, \ldots, z_{k}\right)$ with values in $Z(k)$. By gz we understand the standard action of $G$ on the variables e.g. $g z=\left(z-z^{2} a_{g}\right) / \sigma_{a_{g}}(z), \quad \sigma_{a_{g}}(z)=1-2 a_{g} z+a_{g}^{2} z^{2} \quad$ for special conformal group, $g z=\lambda_{g} \Lambda_{g} z+t_{g}$ for Weyl group.

(ii) The functions $F\left(g, z_{i}, d\right), i=1, \ldots, k$ are analytic in $O\left(z_{1}, \ldots, z_{k}\right)$, where

$F(g, z, d):=\left\{\begin{array}{l}1 \text { for } g \in \text { Poincaré group } \\ \lambda^{d} \text { for dilatation group, } d \geqq 1 \text { (spectral condition) } \\ \left(1 / \sigma_{a_{g}}(z)\right)^{d} \text { for special conformal group, with the } \\ \text { condition }\left.\left(1 / \sigma_{a_{g}}(z)\right)^{d}\right|_{a_{e} \equiv 0}=1 .\end{array}\right.$

Proof. See Appendix 5.

Let us examine for $g \in O\left(z_{1}, \ldots, z_{k}\right)$ the vector

$\Phi(g):=F\left(g, z_{1}, d\right) \ldots F\left(g, z_{k}, d\right) \Psi\left(g z_{1}, \ldots, g z_{k}\right)$.

Since it is a product and composition of analytic functions, it is itself an analytic function in $O\left(z_{1}, \ldots, z_{k}\right)$ with the values in $\mathscr{H}$ (see Appendix 5). Then

Let us choose $g=\exp t X_{a}$, where $X_{a}$ corresponds to $\mathscr{A}$ in the representation.

$$
\Phi\left(\exp t X_{a}\right)=\prod_{i=1}^{k} F\left(\exp t X_{a}, z_{i}, d\right) \Psi\left(\exp t X_{a} z_{1}, \ldots, \exp t X_{a} z_{k}\right)
$$

is an analytic function for $|t|<\varepsilon\left(z_{1}, \ldots, z_{k}\right), \varepsilon\left(z_{1}, \ldots, z_{k}\right)>0$.

Let us now expand this expression in a power series with respect to $t$ (see Appendix 1, Theorem 1). The coefficients of this expansion coincide with the coefficients of the series

$$
\sum_{n=0}^{\infty} \frac{t^{n}}{n !}(i \mathscr{A})^{n} \Psi\left(z_{1}, \ldots, z_{k}\right)
$$

So we conclude that $\Psi\left(z_{1}, \ldots, z_{k}\right)$ is an analytic vector for $\mathscr{A}$. Of course the same holds for linear combinations of $\Psi$. This accomplishes the proof.

Thus from Theorem 3 (Section 1) we have the

Corollary. The symmetrical representation of the Lie algebra of the conformal group on $\mathscr{D}_{z, 0}$, obtained by the extension of the original Lie algebra defined on $\mathscr{D}_{0}$, is uniquely integrable.

Since $\mathscr{D}_{z, 0} \supset \mathscr{D}_{0}$ we have the

Corollary (integrability). The symmetrical representation defined in Section 2 on $\mathscr{D}_{0}$ is integrable to the unitary representation of the conformal group.

We have also from the definition of $\Phi(g)$ (Appendix 5, Lemma 6) the

Corollary. The vectors $\Psi \in \mathscr{D}_{z}$ are analytic vectors for the obtained representation of the conformal group. 
If we denote the generators of the one-parameter subgroups of the obtained representation of the conformal group by $d U\left(X_{a}\right), a=1, \ldots, 15$, we get

$$
\begin{gathered}
d U\left(X_{a}\right) \uparrow \mathscr{D}_{z, 0}=\mathscr{A} \\
d U\left(X_{a}\right) \uparrow \mathscr{D}_{0}=A .
\end{gathered}
$$

So far we were able to show that the symmetrical representation of the Lie algebra on $\mathscr{D}_{z, 0}$ is uniquely integrable. To show that this is also the case for the representation on $\mathscr{D}_{0}$ it is sufficient (see lemma from Section 1) to find a set of generators of this algebra such that each of the elements of this set is essentially selfadjoint on $\mathscr{D}_{0}$.

We choose as our set of generators $\left\{P^{\mu}, M^{\mu \nu}, D, K^{0}\right\}$. It was readily shown that $P^{\mu}, M^{\mu \nu}, D$ are essentially selfadjoint on $\mathscr{D}_{0}$.

Now we show the

Lemma. Operator $K^{0}$ is essentially selfadjoint on $\mathscr{D}_{0}$.

Proof. To the conformal group belongs an element $R$ (the conformal inversion followed by the spatial inversion) so called operator of dimensional reflection ${ }^{1}$, which in the representation yields the unitary equivalence between $d U\left(X_{p^{0}}\right)$ and $d U\left(X_{k^{0}}\right)$ (see [12]). Since $P^{0}$ and $d U\left(X_{p^{0}}\right)$ are positive definite $K^{0}=d U\left(X_{k^{0}}\right) \uparrow \mathscr{D}_{0}$ is also positive definite. On other hand $\mathscr{D}_{0}^{\prime} \subset \mathscr{D}_{0}$ is a total set consisting from semianalytic vectors for $K^{0}$ (see Appendix 2). Hence, using Theorem 2 (Section 1) we conclude that $K^{0}$ is essentially selfadjoint on $\mathscr{D}_{0}$.

Therefore we have the

Corollary (uniqueness). The symmetrical representation of the Lie algebra of the conformal group defined in Section 2 on $\mathscr{D}_{0}$ is uniquely integrable.

Let $U_{R}$ be the operator which corresponds to the dimensional reflection. From the properties of $U_{R}$ and proved before lemmas we have the

Corollary. Each $\Psi \in U_{R} \mathscr{D}_{0}^{\prime}$ is an analytic vector for an arbitrary linear combination of $d U\left(X_{k^{\mu}}\right), d U\left(X_{m^{\mu \nu}}\right), d U\left(X_{\delta}\right)$ which are essentially selfadjoint on $U_{R} \mathscr{D}_{0}$. The vectors of $U_{R^{\prime}} \mathscr{D}_{0}^{\prime}$ are semi-analytic for $d U\left(X_{p^{0}}\right)$ and $d U\left(X_{p^{0}}\right)$ is essentially selfadjoint on $U_{R} \mathscr{D}_{0}$. The symmetrical representation of the Lie algebra of the conformal group on $U_{R} \mathscr{D}_{0}$ obtained by the restriction to $U_{R} \mathscr{D}_{0}, d U(X) \uparrow U_{R} \mathscr{D}_{0}$ is uniquely integrable to the unitary representation of the group.

We see that we have four dense sets: $\mathscr{D}_{0}, \mathscr{D}_{z}, U_{R} \mathscr{D}_{z}, U_{R} \mathscr{D}_{0}$ on which the representation is uniquely integrable.

We do not now, however, whether these sets have common vectors other than $c \Omega$.

We do not know either whether $K^{i}, i=1,2,3$ are essentially selfadjoint on $\mathrm{D}_{0}$ (or equivalently $P^{i}$ on $U_{R} \mathscr{D}_{0}$ ). We know that $U(g) \mathscr{D}_{0} \subset \mathscr{D}_{0}$ for $g \in$ Weyl group but we do not know whether $U(g) \mathscr{D}_{0} \subset \mathscr{D}$ for $g \in$ conformal group. So we do not know also what are the transformation properties of the fields. Since $U(g)$ may lead beyond $\mathscr{D}, U(g) \phi(f) U(g)^{*}$ does not need necessary make sense on $\mathscr{D}_{0}$ or $\mathscr{D}$.

1 The author is greatly indebted to Prof. J. Łopuszański for inspiring discussions and many suggestions concerning the question of the dimensional reflection 
Acknowledgement. The author is grateful to Professor J. Łopuszański for many helpful discussions and critical reading of the manuscript.

The author also thank Professor S. Schlieder for fruitful comments.

\section{Appendix 1. Analytic Functions with Values in Some Banach Space}

Let $\mathbb{E}$ be a field of real $\mathbb{R}$ or complex numbers $\mathbb{C}$. We call on open polydisc $P(a)$ a set

$$
P(a):=\left\{z \in \mathbb{E}^{k} ;\left|z_{i}-a_{i}\right|<r_{i}, 1 \leqq i \leqq k, r_{i}>0\right\} .
$$

Let $O$ be on open set in $\mathbb{E}^{k}$ and $f$ a function on $O$ with values in some Banach space $\mathscr{B}$ over a field $\mathbb{E}$. $f$ is said to be analytic in $O$, if for each point $z_{0} \in O$, there exists an open polydisc $P\left(z_{0}\right)$ contained in $O$ such that in this polydisc

$$
f(z)=\sum_{n=0}^{\infty} \sum_{|v|=n} a_{v}\left(z-z_{0}\right)^{v}, \quad v:=\left(n_{1}, \ldots, n_{k}\right), \quad z^{v}:=z_{1}^{n_{1}} \ldots z_{k}^{n_{k}},
$$

$|v|:=n_{1}+\ldots+n_{k}$, where the right hand side is absolutely convergent in $P\left(z_{0}\right)$ i.e. $\sum_{n=0}^{\infty} \sum_{|v|=n}\left\|a_{v}\right\|\left|z-z_{0}\right|^{v}<\infty$.

Because for absolutely convergent series any series obtained by an arbitrary change of the order of the terms is still absolutely convergent and has the same sum as the given series, the above choice of order is of no importance. So we will write $f(z)=\sum_{v} a_{v}\left(z-z_{0}\right)^{v}$

Let $O$ be an open set in $\mathbb{E}^{k}, f$ a function defined in $O$. We say that the function is differentiable at a point $z_{0} \in O$, if there exists a linear map $D f\left(z_{0}\right)$ of $\mathbb{E}^{k}$ into $\mathscr{B}$ such that:

$$
f(z+h)-f\left(z_{0}\right)=D f\left(z_{0}\right) \cdot h+o(h),
$$

where $\|o(h)\| \leqq \varepsilon\|h\|$ for some $\varepsilon>0$. We have $D f\left(z_{0}\right) \cdot h=\sum_{i=1}^{k} D_{i} f\left(z_{0}\right) h_{i}$ where $D_{i} f\left(z_{0}\right)$ is said a partial derivative at a point $z_{0}$.

We define higher order derivatives inductively as follows

$$
D^{r} f\left(z_{0}\right):=D D^{r-1} f\left(z_{0}\right) \text { if it exists } r \leqq 2,3, \ldots .
$$

So

$$
D^{r} f\left(z_{0}\right) \cdot\left(h^{1}, \ldots, h^{r}\right)=\sum_{1 \leqq j_{1}, \ldots, j_{r} \leqq k} D_{j_{1}}, \ldots, D_{j_{r}} f\left(z_{0}\right) h_{j_{1}}^{1}, \ldots, h_{j_{r}}^{r}, \quad h_{j}^{i} \in \mathbb{E} .
$$

We have

Theorem 1. A function $f$ analytic in an open set $O \subset \mathbb{E}^{k}$ is indefinitely differentiable and all its derivatives are analytic in $O$; furthermore, for each $z_{0} \in O$, there exists a polydisc $P\left(z_{0}\right)$ in which the function is equal to its Taylor series which converges absolutely in this polydisc.

$$
f(z)=\sum_{v} \frac{1}{v !} D^{(v)} f\left(z_{0}\right)\left(z-z_{0}\right)^{v}, \quad D^{(v)}:=D_{1}^{n_{1}} \ldots D_{k}^{n_{k}}, D_{i}^{n}:=\overbrace{D_{i} \ldots D_{i}}^{n}
$$

and $v !:=n_{1} ! \ldots n_{k} !$. We have also $D^{(v)} f\left(z_{0}\right)=a_{v} v !$. 
Theorem 2. Let $O_{1}$ be an open set in $\mathbb{E}^{k}, O_{2}$ an open set in $\mathbb{E}^{q}, g_{i}(1 \leqq i \leqq k) k$ functions analytic in $O_{2}$ with values $\left(g_{1}, \ldots, g_{k}\right)$ in $O_{1}$, and $f$ a function analytic in $O_{1}$ with values in $\mathscr{B}$. Then the composed function $f\left(g_{1}, \ldots, g_{k}\right)$ is defined and analytic in $O_{2}$.

Theorem 3. Let $f, F$ be functions analytic in $O \subset \mathbb{E}^{k}$ with values in $\mathscr{B}$ and $\mathbb{E}$ resp. Then $F \cdot f$ is a function analytic in $O$ with values in $\mathscr{B}$.

Proof. See [13].

\section{Appendix 2}

Lemma 1. Let $T \in \mathscr{S}^{\prime}\left(\mathbb{R}^{4 k}\right)$ and $f_{\{n\}} \in H\left(\mathbb{R}^{4}\right)$ - the space of Hermite functions in four dimensions.

We then have

$$
\left|T\left(f_{\left\{n_{1}\right\}}, \ldots, f_{\left\{n_{k}\right\}}\right)\right| \leqq c\left(1+\left\{n_{1}\right\}\right)^{\left\{p_{1}\right\}} \ldots\left(1+\left\{n_{k}\right\}\right)^{\left\{p_{k}\right\}},
$$

where $(1+\{n\})^{\{p\}}:=\left(1+n_{0}\right)^{p_{0}} \ldots\left(1+n_{3}\right)^{p_{3}}, n_{\mu}, p_{\mu} \in \mathbb{N}, c>0$ depends only on $T$.

Proof. See [14].

Lemma 2. Let $\mathscr{D}_{0}^{\prime}$ be as in Section 3. Let assume further that $A 0,1$ hold and there exists an operator $A$ defined on $\mathscr{D}_{0}$, with the range in $\mathscr{D}$ which satisfies

$$
[A, \phi(f)]=\phi(a f) \text { on } \mathscr{D}_{0}, \quad A \Omega=0,
$$

where $a=P\left(x_{0}, \ldots, x_{3}, \partial^{0}, \ldots, \partial^{3}\right)$ is a polynomial in $x_{\mu}$ and $\partial^{\mu}$ of degree $s$.

If $s \leqq 2$ (resp. $s \leqq 4$ ) then every $\Psi \in \mathscr{D}_{0}^{\prime}$ is an analytic (resp. semi-analytic) vector for $A$.

Proof. We have $A \mathscr{D}_{0} \subset \mathscr{D}_{0}$ and

$$
A^{m} \phi\left(f_{\left\{n_{1}\right\}}\right) \ldots \phi\left(f_{\left\{n_{k}\right\}}\right) \Omega=\sum_{\substack{0 \leqq l_{1}, \ldots, l_{k} \leqq m \\ l_{1}+\ldots+l_{k}=m}} \phi\left(a^{l_{1}} f_{\left\{n_{1}\right\}}\right) \ldots \phi\left(a^{l_{k}} f_{\left\{n_{k}\right\}}\right) \Omega,
$$

hence

$$
\begin{aligned}
& \left\|A^{m} \phi\left(f_{\left\{n_{1}\right\}}\right) \ldots \phi\left(f_{\left\{n_{k}\right\}}\right) \Omega\right\| \\
& \quad \leqq k^{m} \max _{\substack{0 \leqq l_{1}, \ldots, l_{k} \leqq m \\
l_{1}+\ldots+l_{k}=m}}\left\|\phi\left(a^{l_{1}} f_{\left\{n_{1}\right\}}\right) \ldots \phi\left(a^{l_{k}} f_{\left\{n_{k}\right\}}\right) \Omega\right\| .
\end{aligned}
$$

We will look for the estimate of the expressions

$$
\left\|\phi\left(a^{l_{1}} f_{\left\{n_{1}\right\}}\right) \ldots \phi\left(a^{l_{k}} f_{\left\{n_{k}\right\}}\right) \Omega\right\| .
$$

To this end we express $a$ in terms of the annihilation-creation operators

$$
b_{\mu}:=\frac{1}{\sqrt{2}}\left(x_{\mu}+\partial_{\mu}\right), \quad b_{\mu}^{+}:=\frac{1}{\sqrt{2}}\left(x_{\mu}-\partial_{\mu}\right), \quad \mu=0,1,2,3 .
$$

For which

$$
b_{\mu}^{+} f_{\left(\ldots n_{\mu} \ldots\right)}=\left(n_{\mu}+1\right)^{1 / 2} f_{\left(\ldots n_{\mu}+1 \ldots\right)}, \quad b_{\mu} f_{\left(\ldots n_{\mu} \ldots\right)}=n_{\mu}^{1 / 2} f_{\left(\ldots n_{\mu}-1 \ldots\right)} .
$$


Then $a=: W\left(b_{0}^{+}, \ldots, b_{3}\right)$. We have

$$
\begin{aligned}
\| \phi & \left(f_{\left\{n_{1}\right\}}\right) \ldots \phi\left(f_{\left\{n_{k}\right\}}\right) \Omega \| \\
& =\left|\left(\Omega, \phi\left(f_{\left\{n_{k}\right\}}\right) \ldots \phi\left(f_{\left\{n_{1}\right\}}\right) \phi\left(f_{\left\{n_{1}\right\}}\right) \ldots \phi\left(f_{\left\{n_{k}\right\}}\right) \Omega\right)\right|^{1 / 2} \\
& =\left(T\left(f_{\left\{n_{k}\right\}}, \ldots, f_{\left\{n_{1}\right\}}, f_{\left\{n_{1}\right\}}, \ldots, f_{\left\{n_{k}\right\}}\right)\right)^{1 / 2}, \quad \text { where from } A 1 T \in \mathscr{S}^{\prime}\left(\mathbb{R}^{8 k}\right) .
\end{aligned}
$$

So

$$
\begin{aligned}
& \left\|\phi\left(a^{l_{1}} f_{\left\{n_{1}\right\}}\right) \ldots \phi\left(a^{l_{k}} f_{\left\{n_{k}\right\}}\right) \Omega\right\| \\
& \quad=\left(T\left(W^{l_{k}} f_{\left\{n_{k}\right\}}, \ldots, W^{l_{k}} f_{\left\{n_{k}\right\}}\right\}\right)^{1 / 2} \leqq\left(T\left(\hat{W}^{l_{k}} f_{\left\{n_{k}\right\}}, \ldots, \hat{W}^{l_{k}} f_{\left\{n_{k}\right\}}\right)\right)^{1 / 2},
\end{aligned}
$$

where $\hat{W}$ was obtain from $W$ by replacing every coefficient of the polynomial $W$ by the maximum of their absolute values. Let the common coefficient of $\hat{W}$ be $\alpha$. From Lemma 1 and the properties of $b^{+}, b$ we have:

$$
\begin{aligned}
& \left|T\left(\ldots b f_{n} \ldots\right)\right| \leqq c \ldots n^{1 / 2}(1+(n-1))^{p} \ldots, \\
& \left|T\left(\ldots b^{+} f_{n} \ldots\right)\right| \leqq c \ldots(n+1)^{1 / 2}(1+(n+1))^{p} \ldots .
\end{aligned}
$$

Hence we infer that the estimate made according to the Lemma 1 of any expression depending on $b_{\mu}$ and $b_{\mu}^{+}$is not smaller of the corresponding estimate of the quantity constructed by replay of $b_{\mu}$ by $b_{\mu}^{+}$and increasing of the power of $b_{\mu}^{+}$in certain places in the original expression.

In this way we obtain from $\hat{W}$ the sum of monomials of degree $s$ of the form $\alpha\left(\left(b_{0}^{+}\right)^{\frac{1}{s_{0}} \ldots} \ldots\left(b_{3}^{+}\right)^{\frac{1}{s_{3}}}+\ldots+\left(b_{0}^{+}\right)^{s_{0}} \ldots\left(b_{3}^{+}\right)^{s_{3}}\right), \quad$ where $\stackrel{i}{s}_{0}+\ldots+\stackrel{i}{s_{3}}=s$. But

$$
\begin{aligned}
& \left|T\left\{\left[\left(b_{0}^{+}\right)^{\frac{1}{s_{0}}} \ldots\left(b_{3}^{+}\right)^{\frac{1}{s_{3}}}+\ldots+\left(b_{0}^{+}\right)^{s_{0}} \ldots\left(b_{3}^{+}\right)^{r}\right]^{s_{3}} f_{\{n\}}^{l}\right\}\right| \\
& =\mid T\left\{\sum_{u_{1}=0}^{l} \sum_{u_{2}=0}^{u_{1}} \ldots \sum_{u_{r-1}=0}^{u_{r-2}}\left(\begin{array}{l}
l \\
u_{1}
\end{array}\right)\left(\begin{array}{l}
u_{1} \\
u_{2}
\end{array}\right) \ldots\left(\begin{array}{l}
u_{r-2} \\
u_{r-1}
\end{array}\right)\left[\left(b_{0}^{+}\right)^{s_{0}} \ldots\left(b_{3}^{+}\right)^{s_{3}}\right]^{l-u_{1}}\right. \\
& \left.\cdot\left[\left(b_{0}^{+}\right)^{s_{0}} \ldots\left(b_{3}^{+}\right)^{s_{3}}\right]^{u_{1}-u_{2}} \ldots\left[\left(b_{0}^{+}\right)^{s_{0}} \ldots\left(b_{3}^{+}\right)^{s_{3}}\right]^{u_{r-1}} f_{\{n\}}\right\} \\
& =\mid T\left(\sum_{u_{1}=0}^{l} \ldots \sum_{u_{r-1}=0}^{u_{r-2}}\left(\begin{array}{l}
l \\
u_{1}
\end{array}\right) \ldots\left(\begin{array}{l}
u_{r-2} \\
u_{r-1}
\end{array}\right)\left[b_{0}^{+}\right]^{\frac{1}{s_{0}\left(l-u_{1}\right)+\ldots+s_{0} u_{r-1}} \ldots}\right. \\
& \left.\ldots\left[b_{3}^{+}\right]^{\frac{1}{s_{3}\left(l-u_{1}\right)}+\ldots+s_{3} u_{r-1}} f_{\{n\}}\right) \mid \leqq \sum_{u_{1}=0}^{l} \ldots \sum_{u_{r-1}=0}^{u_{r-2}}\left(\begin{array}{l}
l \\
u_{1}
\end{array}\right) \ldots\left(\begin{array}{l}
u_{r-2} \\
u_{r-1}
\end{array}\right) \\
& \cdot \sqrt{\frac{\left[n_{0}+\stackrel{1}{s}_{0}\left(l-u_{1}\right)+\ldots+\stackrel{r}{S}_{0} u_{r-1}\right] !}{n_{0} !}} \ldots \sqrt{\frac{\left[n_{3}+\stackrel{1}{s}_{3}\left(l-u_{1}\right)+\ldots+\stackrel{r}{s_{3}} u_{r-1}\right] !}{n_{3} !}} \\
& \cdot\left|T\left(f_{\left(n_{0}+s_{0}\left(l-u_{1}\right)+\ldots+s_{0} u_{r-1}, \ldots, n_{3}+s_{3}\left(l-u_{1}\right)+\ldots+s_{3} u_{r-1}\right)}\right)\right| \\
& \leqq c \sum_{u_{1}=0}^{l} \ldots \sum_{u_{r-1}=0}^{u_{r-2}}\left(\begin{array}{l}
l \\
u_{1}
\end{array}\right) \ldots\left(\begin{array}{l}
u_{r-2} \\
u_{r-1}
\end{array}\right) \sqrt{\frac{(\bar{n}+s l) !}{\bar{n} !}}\left(1+n_{0}+\stackrel{1}{s}_{0}\left(l-u_{1}\right)+\ldots+\stackrel{r}{s}_{0} u_{r-1}\right)^{p_{0}} \ldots \\
& \ldots\left(1+n_{3}+\stackrel{1}{s}_{3}\left(l-u_{1}\right)+\ldots+\stackrel{r}{s}_{3} u_{r-1}\right)^{p_{3}} \leqq c \sum_{u_{1}=0}^{l} \ldots \sum_{u_{r-1}=0}^{u_{r-2}}\left(\begin{array}{c}
l \\
u_{1}
\end{array}\right) \ldots\left(\begin{array}{l}
u_{r-2} \\
u_{r-1}
\end{array}\right) \sqrt{\frac{(\bar{n}+s l) !}{\bar{n} !}} \\
& \cdot(1+\bar{n}+s l)^{4 \bar{p}}=c r^{l} \sqrt{\frac{(\bar{n}+s l) !}{\bar{n} !}}(1+\bar{n}+s l)^{4 \bar{p}} \text {, }
\end{aligned}
$$


where $\bar{n}=\max \left(n_{0}, \ldots, n_{3}\right), \bar{p}=\max \left(p_{0}, \ldots, p_{3}\right)$.

Hence we have

$$
\begin{aligned}
& \left|T\left(\hat{W}^{l_{k}} f_{\left\{n_{k}\right\}}, \ldots, \hat{W}^{l_{k}} f_{\left\{n_{k}\right\}}\right)\right|^{\frac{1}{2}} \leqq c(r \alpha)^{m} \sqrt{\frac{\left(\bar{n}_{1}+s l_{1}\right) !}{\bar{n}_{1} !} \ldots \sqrt{\frac{\left(\bar{n}_{k}+s l_{k}\right) !}{\bar{n}_{k} !}}} \\
& \quad \cdot\left(1+\bar{n}_{1}+s l_{1}\right)^{4 \bar{p}_{1}} \ldots\left(1+\bar{n}_{k}+s l_{k}\right)^{4 \bar{p}_{k}} \leqq c(r \alpha)^{m} \sqrt{\frac{\left(\left[\bar{n}_{i}\right]_{\max }+m s\right) !}{\left[\bar{n}_{i}\right]_{\max } !}} \\
& \quad \cdot\left(1+\left[\bar{n}_{i}\right]_{\max }+m s\right)^{4 k\left[\bar{p}_{i}\right]_{\max }},
\end{aligned}
$$

where $\left[{ }^{-}\right]_{\max }=\max _{i=1 \ldots k}\left(\cdot_{i}^{-}\right), m=l_{1}+\ldots+l_{k}$.

From this follows

$$
\begin{aligned}
& \left\|A^{m} \phi\left(f_{\left\{n_{1}\right\}}\right) \ldots \phi\left(f_{\left\{n_{k}\right\}}\right) \Omega\right\| \\
& \quad \leqq c(k r \alpha)^{m} \sqrt{\frac{\left(\left[\bar{n}_{i}\right]_{\max }+m s\right) !}{\left[\bar{n}_{i}\right]_{\max } !}}\left(1+\left[\bar{n}_{i}\right]_{\max }+m s\right)^{4 k\left[\bar{p}_{i}\right]_{\max }}
\end{aligned}
$$

and

$$
\begin{aligned}
& \sum_{m=0}^{\infty} \frac{t^{m}}{f(m)}\left\|A^{m} \phi\left(f_{\left\{n_{1}\right\}}\right) \ldots \phi\left(f_{\left\{n_{k}\right\}}\right) \Omega\right\| \\
& \quad \leqq c \sum_{m=0}^{\infty} \frac{(k r \alpha t)^{m}}{f(m)} \sqrt{\frac{\left(\left[\bar{n}_{i}\right]_{\max }+m s\right) !}{\left[\bar{n}_{i}\right]_{\max } !}}\left(1+\left[\bar{n}_{i}\right]_{\max }+m s\right)^{4 k\left[\bar{p}_{i}\right]_{\max }}
\end{aligned}
$$

From d'Alembert criterion the series converges when:

$$
\begin{gathered}
\lim _{m \rightarrow \infty} \frac{r k \alpha t f(m-1)}{f(m)} \sqrt{\left(\left[\bar{n}_{i}\right]_{\max }+m s\right) \ldots\left(\left[\bar{n}_{i}\right]_{\max }+m s-s+1\right)} \\
\cdot\left[\frac{1+\left[\bar{n}_{i}\right]_{\max }+m s}{1+\left[\bar{n}_{i}\right]_{\max }+(m-1) s}\right]^{4 k\left[\bar{p}_{i}\right]_{\max }}<1 .
\end{gathered}
$$

Thus

(i) in the case of analytic vectors $f(m)=m$ !

$r k \alpha t \lim _{m \rightarrow \infty} \frac{1}{m} \sqrt{\left(\left[\bar{n}_{i}\right]_{\max }+m s\right) \ldots\left(\left[\bar{n}_{i}\right]_{\max }+m s-s+1\right)}<1$.

When $s \leqq 2$ it is satisfied for $t<\frac{1}{2 k \alpha r}$.

(ii) In the case of semi-analytic vectors $f(m)=(2 m)$ !

$r k \alpha t \lim _{m \rightarrow \infty} \frac{1}{2 m(2 m-1)} \sqrt{\left(\left[\bar{n}_{i}\right]_{\max }+m s\right) \ldots\left(\left[\bar{n}_{i}\right]_{\max }+m s-s+1\right)}<1$.

When $s \leqq 4$ it is satisfied for $t<\frac{1}{4 k \alpha r}$. This concludes the proof. 
In particular e.g., for

$$
\begin{aligned}
A=D, & a=\delta=i\left(4-d+x^{\mu} \partial_{\mu}\right)=i\left[5-d+{ }_{2}\left(b^{2}-\left(b^{+}\right)^{2}\right)\right]=W\left(b_{0}^{+}, \ldots, b_{3}\right) \\
& \alpha=\max \left(|5-d|, \frac{1}{2}\right), \quad r=4, \quad s=2, \\
A= & K_{0}, \quad a=k_{0}=i\left[2(4-d) x_{0}+2 x_{0} x^{\mu} \partial_{\mu}-x^{2} \partial_{0}\right] \\
= & \frac{i}{\sqrt{2}}\left[-\frac{1}{2} b_{0}^{+}\left(b^{+}\right)^{2}-\frac{1}{2}\left(b_{0}^{+}\right)^{2} b_{0}-b_{0}^{+}\left(\vec{b}^{+} \vec{b}\right)+\frac{3}{2}\left(\vec{b}^{+}\right)^{2} b_{0}+\frac{1}{2} b_{0}^{+} b_{0}^{2}-\frac{3}{2} b_{0}^{+}(\vec{b})^{2}\right. \\
& \left.+\left(\vec{b}^{+} \vec{b}\right) b_{0}+2(4-d) b_{0}^{+}+\frac{1}{2} b_{0} b^{2}+2(6-d) b_{0}\right]=W\left(b_{0}^{+}, \ldots, b_{3}\right), \\
\alpha= & \sqrt{2} \max (|4-d|,|6-d|), \quad r=4, \quad s=3 .
\end{aligned}
$$

\section{Appendix 3}

Lemma. If the Axioms A0,1,2,3'a,c hold and there exists an operator $A$ defined and symmetrical on $\mathscr{D}_{0}$ with the range in $\mathscr{D}$ satisfying

$$
[A, \phi(f)]=\phi(a f) \quad \text { on } \mathscr{D}_{0}, \quad A \Omega=0,
$$

where $a=P\left(x^{0}, \ldots, x^{3}, \partial_{0}, \ldots, \partial_{3}\right)$-polynomial in $x^{\mu}, \partial_{\mu}$, then there exists an operator $\mathscr{A}$ defined and symmetrical on the domain $\mathscr{D}_{z, 0} \supset \mathscr{D}_{0}$ (for definition see below) such that $\mathscr{A} \mathscr{D}_{z, 0} \subset \mathscr{D}_{z, 0}, \mathscr{A} \uparrow \mathscr{D}_{0}=A$.

Proof. Let $\mathscr{W}_{n}\left(f_{1}, \ldots, f_{n}\right):=\left(\Omega, \phi\left(f_{1}\right) \ldots \phi\left(f_{n}\right) \Omega\right)$. From $A 1$, the Nuclear Theorem [16], as well as that $\otimes^{n} \mathscr{S}\left(\mathbb{R}^{4}\right)$ is dense in $\mathscr{S}\left(\mathbb{R}^{4 n}\right)$, follows the existence of $\mathscr{W}_{n}(f) \in \mathscr{S}^{\prime}\left(\mathbb{R}^{4 n}\right) \quad$ such that $\mathscr{W}_{n}\left(f_{1} \otimes \ldots \otimes f_{n}\right)=\mathscr{W}_{n}\left(f_{1}, \ldots, f_{n}\right)$ and $\mathscr{W}_{n}(f)$ $=\lim \mathscr{W}_{n}\left(f_{1}, \ldots, f_{\mathfrak{n}}\right)$ for $f_{1} \otimes \ldots \otimes f_{n} \rightarrow f$ in the topology of $\mathscr{S}\left(\mathbb{R}^{4 n}\right)$.

Moreover there exists the vector valued distribution $\Psi(f)$ such that $\Psi\left(f_{1} \otimes \ldots \otimes f_{n}\right)=\phi\left(f_{1}\right) \ldots \phi\left(f_{n}\right) \Omega$ and

$$
\Psi(f)=\|\|-\lim \phi\left(f_{1}\right) \ldots \phi\left(f_{n}\right) \Omega \quad \text { for } \quad f_{1} \otimes \ldots \otimes f_{n} \stackrel{\mathscr{P}\left(\mathbb{R}^{4 n}\right)}{\longrightarrow} f .
$$

From $A 1$

$$
\mathscr{W}_{l+k}\left(\bar{g}_{l}, \ldots, \bar{g}_{1}, f_{1}, \ldots, f_{k}\right)=\left(\phi\left(g_{1}\right) \ldots \phi\left(g_{l}\right) \Omega, \phi\left(f_{1}\right) \ldots \phi\left(f_{k}\right) \Omega\right) .
$$

Hence

$$
\begin{aligned}
& \left(A \phi\left(g_{1}\right) \ldots \phi\left(g_{l}\right) \Omega, \phi\left(f_{1}\right) \ldots \phi\left(f_{k}\right) \Omega\right) \\
& \quad=\sum_{i=1}^{l} \mathscr{W}_{l+k}\left(\bar{g}_{l}, \ldots, \overline{a g}_{i}, \ldots, g_{1}, f_{1}, \ldots, f_{k}\right) \\
& =\left(\phi\left(g_{1}\right) \ldots \phi\left(g_{l}\right) \Omega, A \phi\left(f_{1}\right) \ldots \phi\left(f_{k}\right) \Omega\right) \\
& \quad=\sum_{j=1}^{k} \mathscr{W}_{l+k}\left(\bar{g}_{l}, \ldots, \bar{g}_{1}, f_{1}, \ldots, a f_{j}, \ldots, f_{k}\right) .
\end{aligned}
$$


Taking the limit we obtain

$$
\begin{aligned}
& \mathscr{W}_{l+k}\left(g^{+}, f\right)=(\Psi(g), \Psi(f)), \\
& \mathscr{W}_{l+k}\left(\left(\sum_{i=1}^{l} a_{i} g\right)^{+}, f\right)=\mathscr{W}_{l+k}\left(g^{+}, \sum_{j=1}^{k} a_{j} f\right),
\end{aligned}
$$

where

$$
g^{+}\left(x_{1}, \ldots, x_{l}\right):=\overline{g\left(x_{l}, \ldots, x_{1}\right)}
$$

\section{because}

$$
f_{1} \otimes \ldots a f_{i} \otimes \ldots f_{l} \stackrel{\mathscr{S}\left(\mathbb{R}^{4 l}\right)}{\longrightarrow} a_{i} f \quad \text { when } \quad f_{1} \otimes \ldots \otimes f_{l} \stackrel{\mathscr{S}\left(\mathbb{R}^{4 l}\right)}{\longrightarrow} f,
$$

where

$$
a_{i}=P\left(x_{i}^{0}, \ldots, x_{i}^{3}, \partial_{0}^{i}, \ldots, \partial_{3}^{i}\right) .
$$

Hence

$$
\left(\Psi\left(\sum_{i=1}^{l} a_{i} g\right), \Psi(f)\right)=\left(\Psi(g), \Psi\left(\sum_{j=1}^{k} a_{j} f\right)\right)
$$

Let $Q_{l}, R_{k}$ be polynomial operators in $x_{i}^{\mu}, \partial_{\mu}^{i}$. Since

$$
Q_{l} \mathscr{S}\left(\mathbb{R}^{4 l}\right) \subset \mathscr{S}\left(\mathbb{R}^{4 l}\right), \quad R_{k} \mathscr{S}\left(\mathbb{R}^{4 k}\right) \subset \mathscr{S}\left(\mathbb{R}^{4 k}\right),
$$

hence

$$
\begin{aligned}
& \mathscr{W}_{l+k}\left(\left(\sum_{i=1}^{l} a_{i} Q_{l} g\right)^{+}, R_{k} f\right)=\mathscr{W}_{l+k}\left(\left(Q_{l} g\right)^{+}, \sum_{j=1}^{k} a_{j} R_{k} f\right), \\
& \left(\Psi\left(\sum_{i=1}^{l} a_{i} Q_{l} g\right), \Psi\left(R_{k} f\right)\right)=\left(\Psi\left(Q_{l} g\right), \Psi\left(\sum_{j=1}^{k} a_{j} R_{k} f\right)\right) .
\end{aligned}
$$

Now we use the spectral condition.

From $A 3^{\prime} a, c, \mathscr{W}_{n}(f)$ is a boundary value of a function $\mathscr{W}_{n}\left(z_{1}, \ldots, z_{n}\right)$ defined and analytic in the domain $\left\{\left(z_{1}, \ldots, z_{n}\right) ; \operatorname{Im}\left(z_{i+1}-z_{i}\right) \in V^{+}, i=1, \ldots, n-1\right\}$ i.e.,

$$
\mathscr{W}_{n}(f)=\lim _{\substack{\lambda \rightarrow 0 \\ \lambda>0}} \int \mathscr{W}_{n}\left(x_{1}+i \lambda y_{1}, \ldots, x_{n}+i \lambda y_{n}\right) f\left(x_{1}, \ldots, x_{n}\right) d^{4} x_{1} \ldots d^{4} x_{n} .
$$

Proof. See [15].

In a similar way $\Psi(f)$ is a boundary value of a vector valued function $\Psi\left(z_{1}, \ldots, z_{n}\right)$, said "an extension" of $\Psi(f)$, defined and analytic in the domain

$$
Z(n):=\left\{\left(z_{1}, \ldots, z_{n}\right) ; \operatorname{Im} z_{1} \in V^{+}, \operatorname{Im}\left(z_{i+1}-z_{i}\right) \in V^{+}, i=1, \ldots, n-1\right\}
$$

i.e.,

$$
\Psi(f)=\|\|-\lim _{2 \rightarrow n} \int \Psi\left(x_{1}+i \lambda y_{1}, \ldots, x_{n}+i \lambda y_{n}\right) f\left(x_{1}, \ldots, x_{n}\right) d^{4} x_{1} \ldots d^{4} x_{n}
$$


and

$$
\begin{aligned}
& \mathscr{W}_{l+k}\left(\bar{\omega}_{l}, \ldots, \bar{\omega}_{1}, z_{1}, \ldots, z_{k}\right)=\left(\Psi\left(\omega_{1}, \ldots, \omega_{l}\right), \Psi\left(z_{1}, \ldots, z_{k}\right)\right) \text { for } \\
& \left(z_{1}, \ldots, z_{k}\right) \in Z(k),\left(\omega_{1}, \ldots, \omega_{l}\right) \in Z(l) \quad \text { see }[16,17] .
\end{aligned}
$$

We may consider the last equality as an analytic continuation of the equality (1). Similarly, by continuation the equalities (2), $\left(2^{\prime}\right)$ we obtain for $\left(\omega_{1}, \ldots, \omega_{l}, z_{1}, \ldots, z_{k}\right) \in Z(l) \times Z(k)$

$$
\begin{aligned}
& \left(\sum_{i=1}^{l} \tilde{a}_{i} \Psi\left(\omega_{1}, \ldots, \omega_{1}\right), \Psi\left(z_{1}, \ldots, z_{k}\right)\right) \\
& \quad=\left(\Psi\left(\omega_{1}, \ldots, \omega_{l}\right), \sum_{j=1}^{k} \tilde{a}_{j} \Psi\left(z_{1}, \ldots, z_{k}\right)\right),
\end{aligned}
$$

where $\tilde{a}_{i}$ are defined by the following equality: for each

$$
f, g \in \mathscr{S}\left(\mathbb{R}^{4}\right), \int f(x)(a g)(x) d^{4} x=\int(\tilde{a} f)(x) g(x) d^{4} x \text { and } \tilde{a}_{i}=\left.\tilde{a}\right|_{x=z_{i}} .
$$

Finally, from (3), $\left(3^{\prime}\right)$ we obtain

$$
\begin{aligned}
& \left(\tilde{Q}_{l}\left(\sum_{i=1}^{l} \tilde{a}_{i} \Psi\left(\omega_{1}, \ldots, \omega_{l}\right)\right), \tilde{R}_{k} \Psi\left(z_{1}, \ldots, z_{k}\right)\right) \\
& \quad=\left(\tilde{Q}_{l} \Psi\left(\omega_{1}, \ldots, \omega_{l}\right), \tilde{R}_{k}\left(\sum_{j=1}^{k} \tilde{a}_{j}\right) \Psi\left(z_{1}, \ldots, z_{k}\right)\right),
\end{aligned}
$$

where $\tilde{Q}_{l}$ is defined by the equality: for each $f, g \in \mathscr{S}\left(\mathbb{R}^{4 l}\right)$,

$$
\begin{aligned}
& \int f\left(x_{1}, \ldots, x_{l}\right)\left(Q_{l} g\right)\left(x_{1}, \ldots, x_{l}\right) d^{4} x_{1} \ldots d^{4} x_{l} \\
& \quad=\int\left(\tilde{Q}_{l} f\right)\left(x_{1}, \ldots, x_{l}\right) g\left(x_{1}, \ldots, x_{l}\right) d^{4} x_{1} \ldots d^{4} x_{l}
\end{aligned}
$$

and substitution $x \rightarrow \omega$. Similarly we define $\tilde{R}_{k}$.

Taking the limit with $\tilde{R}_{k} \equiv 1\left(\tilde{Q}_{l} \equiv 1\right)$ we obtain

$$
\begin{aligned}
& \left(\tilde{Q}_{l}\left(\sum_{i=1}^{l} \tilde{a}_{i}\right) \Psi\left(\omega_{1}, \ldots, \omega_{l}\right), \Psi(h)\right)=\left(\tilde{Q}_{l} \Psi\left(\omega_{1}, \ldots, \omega_{l}\right), \Psi\left(\sum_{j=1}^{k} a_{j} h\right)\right), \\
& \left(\Psi\left(\sum_{i=1}^{l} a_{i} f\right), \tilde{R}_{k} \Psi\left(z_{1}, \ldots, z_{k}\right)\right) \\
& \quad=\left(\Psi(f), \tilde{R}_{k}\left(\sum_{j=1}^{k} \tilde{a}_{j}\right) \Psi\left(z_{1}, \ldots, z_{k}\right)\right),
\end{aligned}
$$

for $\left(\omega_{1}, \ldots, \omega_{l}\right) \in Z(l),\left(z_{1}, \ldots, z_{k}\right) \in Z(k), h \in \mathscr{S}\left(\mathbb{R}^{4 k}\right), f \in \mathscr{S}\left(\mathbb{R}^{4 l}\right)$. Now we define $\mathscr{D}_{z}:=\operatorname{Lin}\left\{\tilde{Q}_{l} \Psi\left(\omega_{1}, \ldots, \omega_{l}\right) ;\left(\omega_{1}, \ldots, \omega_{l}\right) \in Z(l), \Omega\right.$ for $l=0, \tilde{Q}_{l}$ is an arbitrary polynomial operator in $\omega, \frac{\partial}{\partial \omega}, l=0,1, \ldots, \Psi$ is "an extension" of $\left.\Psi(f)\right\}$.

The set $\mathscr{D}_{z}$ is dense in $\mathscr{H}$. This follows from two facts: (i) $\mathscr{D}_{0}$ is dense in $\mathscr{H}$, (ii) every $\Psi \in \mathscr{D}_{0}$ is the limit of the vectors from $\mathscr{D}_{z}$. 
We have also

$\mathscr{D}_{z}=\operatorname{Lin}\left\{\Psi\left(\omega_{1}, \ldots, \omega_{l}\right) ;\left(\omega_{1}, \ldots, \omega_{l}\right) \in Z(l), \Omega\right.$ for $l=0, \Psi$ is "an extension" of $\bar{\Psi}(f):=\Psi\left(Q_{l} f\right), Q_{l}$ is an arbitrary polynomial operator in $\left.x, \frac{\partial}{\partial x}, l=0,1, \ldots\right\}$.

Let $\mathscr{D}_{z, 0}$ be defined by

$$
\mathscr{D}_{z ; 0}:=\operatorname{Lin}\left(\left\{\Psi(f) ; f \in \mathscr{S}\left(R^{4 k}\right), k=1,2 \ldots\right\} \cup \mathscr{D}_{z}\right) .
$$

Of course $\mathscr{D}_{z, 0} \supset \mathscr{D}_{0}$.

We define the operator $\mathscr{A}$ on $\mathscr{D}_{z, 0}$ by means of the equalities

$$
\begin{aligned}
& \mathscr{A} \Psi(f):=\Psi\left(\sum_{j=1}^{k} a_{j} f\right), \quad \mathscr{A} \Omega:=0, \\
& \mathscr{A} \tilde{Q}_{l} \Psi\left(\omega_{1}, \ldots, \omega_{l}\right):=\tilde{Q}_{l} \sum_{i=1}^{l} \tilde{a}_{i} \Psi\left(\omega_{1}, \ldots, \omega_{l}\right) .
\end{aligned}
$$

We shall show that the definition above is meaningful and that $\mathscr{A}$ is symmetrical on $\mathscr{D}_{z, 0}$.

Let $\xi, \zeta \in \mathscr{D}_{z, 0}$. From the definition $\mathscr{D}_{z, 0}$ we have (finite sum)

$$
\begin{aligned}
& \xi=\sum_{f, k} \Psi(f)+\sum_{\tilde{Q}_{l},\left(\omega_{1}, \ldots, \omega_{l}\right) \in Z(l), l} \tilde{Q}_{l} \Psi\left(\omega_{1}, \ldots, \omega_{l}\right), \\
& \zeta=\sum_{h, l} \Psi(h)+\tilde{R}_{\tilde{R}_{k},\left(z_{1}, \ldots, z_{k}\right) \in Z(k), k} \tilde{R}_{k} \Psi\left(z_{1}, \ldots, z_{k}\right),
\end{aligned}
$$

and

$$
\begin{aligned}
& \mathscr{A} \xi=\sum_{f, k} \Psi\left(\sum_{j=1}^{k} a_{j} f\right)+\sum_{\left.\tilde{Q}_{l,\left(\omega_{1}, \ldots, \omega\right.}\right) \in Z(l), l} \tilde{Q}_{l} \sum_{i=1}^{l} \tilde{a}_{i} \Psi\left(\omega_{1}, \ldots, \omega_{l}\right), \\
& \mathscr{A} \zeta=\sum_{h, l} \Psi\left(\sum_{i=1}^{l} a_{i} h\right)+\sum_{\tilde{R}_{k},\left(z_{1}, \ldots, z_{k}\right) \in Z(k), k} \tilde{R}_{k} \sum_{j=1}^{k} \tilde{a}_{j} \Psi\left(z_{1}, \ldots, z_{k}\right) .
\end{aligned}
$$

From the equalities $\left(2^{\prime}\right),(4-6)$ we have

$$
(\mathscr{A} \xi, \zeta)=(\xi, \mathscr{A} \zeta)
$$

Thus, if $\xi=0$ then $\mathscr{A} \xi=0$, because $\mathscr{D}_{z, 0}$ is dense in $\mathscr{H}$. Hence the definition is correct and the operator is symmetrical.

In a straightforward manner we conclude also from the definition that $\mathscr{A} \uparrow \mathscr{D}_{0}=A$.

This concludes the proof.

\section{Appendix 4}

The aim of this Appendix is to exhibit some essential differences between Theorem 3 from Section 1 and Lemma 9.1 from Nelson [6]. 
Let $T$ be a Lie algebra of symmetrical operators defined on a common invariant dense domain $\mathscr{D} \subset \mathscr{H}$.

We say:

(1) A vector $\Psi \in \mathscr{D}$ is analytic for the whole Lie algebra, if for $0 \geqq t<t_{\Psi}^{(1)}$ and some basis $A_{1}, \ldots, A_{n}$ of the Lie algebra, the series

$$
\sum_{k=0}^{\infty} \frac{1}{k !} \sum_{1 \leqq a_{1}, \ldots, a_{k} \leqq n} t^{k} A_{a_{1}} \ldots A_{a_{k}} \Psi
$$

is absolutely convergent.

(2) A vector $\Psi \in \mathscr{D}$ is analytic for $A_{1}, \ldots, A_{n}$ separately, if it is analytic for every element $A_{a}$ in a given basis, $a=1, \ldots, n$ i.e., that the series $\sum_{k=0}^{\infty} \frac{t^{k}}{k !} A_{a}^{k} \Psi$ ( $n$ finite) are absolutely convergent for $0 \leqq t<t_{\Psi}^{(2)}, a=1, \ldots, n$.

We have of course $t_{\Psi}^{(1)} \leqq t_{\Psi}^{(2)}$.

Let $T^{(1)},\left(T^{(2)}\right)$ be a set consisting of analytic vectors for the whole Lie algebra (for $A_{1}, \ldots, A_{n}$ separately).

We define $\inf _{\Psi \in T^{(1)}} t_{\Psi}^{(1)}=: t_{T^{(1)}}^{(1)},\left(t_{T^{(2)}}^{(2)}:=\inf _{\Psi_{\in} T^{(2)}} t_{\Psi}^{(2)}\right)$.

It can happen that for some $T^{(2)}, t_{T^{(2)}}^{(2)}=0$.

In the proof of Lemma 9.1 Nelson assumes that there exists a dense invariant set $T^{(1)} \subset \mathscr{D}$ such that $t_{T^{(1)}}^{(1)}>0$ (because he exploits in the proof the Campbell-Hausdorff formula). The criterion given by Theorem 3 is more general. It is sufficient to assume that there exists a dense (or total) set $T^{(2)} \subset \mathscr{D}$.

The method of the proof of the Theorem 3 is different from that used in the Lemma 9.1 of Nelson and more general. For details see $[8,9]$.

\section{Appendix 5}

Lemma 1. $z^{d}:=e^{d \log z}$, where $\log 1=0, d \in \mathbb{C}$ is an analytic function of $z \in \mathbb{C}$ in the complement in $\mathbb{C}$ of the half-line $(-\infty, 0]$.

Proof. See [18].

Let $\sigma_{a}(\omega):=1-2 a \omega+a^{2} \omega^{2}, \omega, a \in \mathbb{C}^{4}, a \omega:=a_{0} \omega_{0}-\vec{a} \omega, a^{2}:=a a$.

Lemma 2. For every fixed $z \in \mathbb{C}^{4}$ there exist an open set $A(z) \subset \mathbb{C}^{4}$ containing zero and an open set $B(z)$ containing $z$ such that $\left(\sigma_{a}(\omega)\right)^{d},\left(\sigma_{0}(\omega)\right)^{d}=1$ is the analytic function in $(a, \omega) \in A(z) \times B(z) \subset \mathbb{C}^{8}$.

Proof. $\sigma_{a}(\omega)$ is analytic in $\mathbb{C}^{8}$. Since $\sigma_{0}(\omega)=1$ there exists $A(z)$ and $B(z)$ such that $\sigma_{a}(\omega) \in \mathbb{C} \backslash(-\infty, 0]$ for $(a, \omega) \in A(z) \times B(z)$. Using Theorem 2 (Appendix 1) and Lemma 1 we have that $\left(\sigma_{a}(\omega)\right)^{d},\left(\sigma_{0}(\omega)\right)^{d}=1$ is analytic in $A(z) \times B(z)$.

Corollary. $\left(\omega-a \omega^{2}\right) / \sigma_{a}(\omega)$ is an analytic function with values in $\mathbb{C}^{4}$ for $\omega \in B(z)$ and $a \in A(z)$. 
Lemma 3. Any group element $g$ of the conformal group has the canonical decomposition in terms of translations $g_{T}$, special conformal transformations $g_{k}$, special Lorentz transformations $g_{A}$ and dilatations $g_{\lambda} ; g=g_{T} g_{k} g_{A} g_{\lambda}$.

Proof. See [1].

Thus, if $O$ is some open neighbourhood of the unity in the conformal group, then there exist an open neighbourhood of the unity in the translation group $O_{T}$, special conformal group $O_{k}$, Lorentz group $O_{\Lambda}$, dilatation group $O_{\lambda}$ such that $O=O_{T} \cdot O_{k}$ $\cdot O_{\Lambda} \cdot O_{\lambda}$.

Lemma 4. gz can be considered on the complex (or real) Minkowski space as a local Lie group of local transformations. In other words, for every $z \in \mathbb{C}^{4}$ there exists an open neighbourhood $O(z)$ of the unity, for which $g z$ is defined, analytic in $O(z)$ and $g_{1}\left(g_{2} z\right)=\left(g_{1} g_{2}\right) z$ if $g_{1}, g_{2}, g_{1} g_{2} \in O(z)$.

Proof. Let $z$ be fixed. $g z=g_{T} g_{k} g_{\lambda} g_{\lambda} z$. It is known that for the Weyl group $g z$ is analytic. Now we take for $O(z)$ such an open set for which $O_{\Lambda}(z) O_{\lambda}(z) z \subset B(z)$ and $O_{k}(z) \subset A(z)$, where $O(z)=O_{T}(z) O_{k}(z) O_{\Lambda}(z) O_{\lambda}(z), A(z), B(z)$ as in Lemma 2. Using Theorem 2 in Appendix 1 and Corollary we obtain that $g z$ is analytic in $O(z)$. By straightforward calculation one can check that also $g_{1}\left(g_{2} z\right)=\left(g_{1} g_{2}\right) z$ if $g_{1}, g_{2}, g_{1} g_{2} \in O(z)$.

\section{Remark}

From the definition of $g z$ it is clear that it is singular on the Minkowski space. A way out of this problem is to compactify the usual Minkowski space by adding points at infinity (see [19-21]).

On this compactified Minkowski space the conformal group can act as a well defined group of transformations. But in this case only a local causal structure can be defined [21]. This structure can be lifted to a global causal structure, if one considers the universal covering space of compactified Minkowski space. On this space acts the universal covering group of the Minkowskian conformal group $[22,23]$.

Lemma 5. Let $O(z)$ be as in proof of Lemma 4. Then for fixed $z$

$$
F(g, z, d):=\left[\frac{1}{\sigma_{a_{g_{k}}}\left(g_{\lambda} g_{\lambda} z\right)}\right]^{d}
$$

is analytic for $g \in O(z)$.

Proof. This follows immediately from Lemma 2.

Lemma 6. There exists an open neighbourhood $O\left(z_{1}, \ldots, z_{k}\right)$ of the unity for which $\Phi(g)$ defined as in Section 3 is analytic in $O\left(z_{1}, \ldots, z_{k}\right)$.

Proof. This follows from Theorem 2, and 3 in Appendix 1 for

$$
O\left(z_{1}, \ldots, z_{k}\right):=\left\{g \in \bigcap_{i=1}^{k} O\left(z_{i}\right) ;\left(g z_{1}, \ldots, g z_{k}\right) \in Z(k)\right\} .
$$




\section{References}

1. Hortaçsu,M., Seiler, R., Schroer,B.: Phys. Rev. D5, 2519 (1972)

2. Rühl,W.: Commun. math. Phys. 27, 53 (1972); 30, 287 (1973); 34, 149 (1973)

3. Schroer, B., Swieca,J. A.: Phys. Rev. D10, 480 (1974)

4. Swieca,J. A., Völkel, A.H.: Commun. math. Phys. 29, 319 (1973)

5. Lüscher,M., Mack,G.: Commun. math. Phys. 41, 203 (1975)

6. Nelson, E.: Ann. Math. 70, 372 (1959)

7. Simon, B.: Indian J. Math. 20, 1145 (1971)

8. Flato, M., Simon,J., Snellman,H., Sternheimer,D.: Ann. Scient. Éc. Norm. Sup. 5, 423 (1972)

Simon,J.: Comm. math. Phys. 28, 39 (1972)

9. Flato, M., Simon,J.: J. Funct. Anal. 13, 268 (1973)

10. Schaaf,M.: Rep. Math. Phys. 4, 275 (1973)

11. Snellman, M.: J. Math. Phys. 15, 1054 (1974)

12. Mack, G.: Desy preprint, December 1975

13. Dieudonné,J.: Foundations of modern analysis. New York: Academic Press 1969

14. Simon, B.: J. Math. Phys. 12, 140 (1971)

15. Bros,J., Epstein,H., Glaser,V.: Commun. math. Phys. 6, 77 (1967)

16. Jost, R.: The general theory of quantized fields, Providence, Rhode Island: American Mathematical Society 1965

17. Osterwalder, K.: In: Constructive quantum field theory, (eds. G. Velo, A. S. Wightman). Lecture Notes in Physics 25. Berlin-Heidelberg-New York: Springer 1973

18. Dieudonné,J.: Infinitesimal calculus. Herman: Paris 1971

19. Dirac,P.: Ann. Math. 37, 657 (1936)

20. Penrose, R.: Relativistic symmetry groups. In: Group theory in non-linear problems (ed. A. O. Barut). Dordrecht: D. Reidel Publishing Company 1974

21. Go,T.H., Kastrup,H.A., Mayer,D.H.: Rep. Math. Phys. 5, 43 (1974)

22. Go,T.H.: Commun. math. Phys. 41, 157 (1975)

23. Segal,I.E.: Preprint, MIT, Cambridge: Massachusetts (1974)

Communicated by R. Haag

Received December 1, 1976 